\title{
Topical treatment options for conjunctival neoplasms
}

\author{
Jonathan W Kim \\ David H Abramson
}

Ophthalmic Oncology Service, Memorial Sloan-Kettering Cancer Center, New York, NY, USA
Correspondence: Jonathan W Kim

Ophthalmic Oncology Service, Memorial Sloan-Kettering Cancer Center, 1275 York Ave, New York, NY I002 I, USA

Tel + I 2126397232

Email kimj12@mskcc.org

\begin{abstract}
Topical therapies offer a nonsurgical method for treating conjunctival tumors by delivering high drug concentrations to the ocular surface. Over the past ten years, topical agents have been used by investigators to treat various premalignant and malignant lesions of the conjunctiva, such as primary acquired melanosis with atypia, conjunctival melanoma, squamous intraepithelial neoplasia and squamous cell carcinoma of the conjunctiva, and pagetoid spread of the conjunctiva arising from sebaceous cell carcinoma. Despite the enthusiasm generated by the success of these agents, there are unanswered questions regarding the clinical efficacy of this new nonsurgical approach, and whether a single topical agent can achieve cure rates comparable with traditional therapies. Furthermore, the long-term consequences of prolonged courses of topical chemotherapeutic drugs on the ocular surface are unknown, and the ideal regimen for each of these agents is still being refined. In this review, we present specific guidelines for treating both melanocytic and squamous neoplasms of the conjunctiva, utilizing the available data in the literature as well as our own clinical experience at the Memorial Sloan-Kettering Cancer Center. Keywords: topical therapies, conjunctival neoplasms melanosis, Mitomycin-C, 5-Fluorouracil
\end{abstract}

\section{Introduction}

Historically, the established method of treatment for conjunctival neoplasms has been wide local excision, with or without supplemental cryotherapy to the surgical margins. Unfortunately, conjunctival neoplasms can be diffuse or multifocal, with borders that are difficult to detect clinically or even with histopathologic examination. Topical therapies offer a nonsurgical method for treating the entire conjunctival surface with less dependence on defining tumor margins. Topical administration can also deliver high drug concentrations to the ocular surface with negligible systemic side effects. Unlike surgical excision, topical treatments can be titrated and even repeated according to the clinical response. The cost is typically minimal when compared with surgical intervention, and there is little risk of spreading neoplastic cells with topical therapy. Because of these potential advantages, there has been growing interest in the use of topical agents for various conjunctival neoplasms.

Over the past ten years, topical agents have been used by investigators to treat various premalignant and malignant lesions of the conjunctiva, such as primary acquired melanosis with atypia, conjunctival melanoma, squamous intraepithelial neoplasia and squamous cell carcinoma of the conjunctiva, and pagetoid spread of the conjunctiva arising from sebaceous cell carcinoma. Despite the enthusiasm generated by the success of these agents, it should be kept in mind that chemotherapeutic drugs can be potentially toxic to the ocular surface. There are also issues with compliance that do not exist with traditional therapies. The long-term consequences of prolonged courses of topical chemotherapeutic agents on the ocular surface are unknown, and the ideal regimen for each of these agents is still being refined. Perhaps most importantly, there are unanswered questions regarding the clinical efficacy of this new nonsurgical 
approach, and whether a single topical agent can achieve long-term cures of conjunctival neoplasms.

The goal of this review is to summarize the essential published literature on the use of topical agents for both melanocytic and squamous conjunctival neoplasms. Furthermore, our aim is to offer specific therapeutic guidelines for clinicians using the available data that has been published by various investigators, augmented by our own clinical experience at the Memorial Sloan-Kettering Cancer Center in New York, NY. To accomplish these goals, we reviewed the relevant medical literature by searching MEDLINE (1970-2006) for articles describing the use of topical chemotherapy agents for conjunctival neoplastic processes. In performing this search, the following keywords were used both singly and in combination: chemotherapy, topical, mitomycin, 5-fluorouracil, interferon, conjunctival neoplasm, conjunctival tumor, primary acquired melanosis (PAM), conjunctival melanoma, squamous cell carcinoma, ocular surface squamous neoplasia, conjunctival papilloma, and sebaceous cell carcinoma. Additional articles were identified by reviewing the bibliographies of publications found in the MEDLINE search.

\section{Topical agents for conjunctival neoplasms \\ Mitomycin-C}

Mitomycin C (MMC) is an alkylating agent that binds to DNA during all phases of the cell cycle, leading to irreversible cross-linking and inhibition of nucleotide synthesis. Isolated from Strepmyces caespitosus, enzymatic reduction of its quinine ring and methoxy group permits MMC to cross link DNA between adenine and guanine at three different sites (McKelvie and Daniell 2001). Under aerobic conditions, intermediates of MMC also react with molecular oxygen to generate free radicals, causing cytotoxicity via lipid peroxidation. Hence, MMC appears to be toxic to both proliferating and nonproliferating cells. In clinical oncology, MMC has been used extensively to treat superficial transitional cell carcinoma of the bladder epithelium (Murphy, Soloway et al 1981). In its ophthalmic use, MMC has an established track record for safety and efficacy in pterygium and glaucoma surgery (Palmer 1991; Donnenfeld et al 2003). When applied to conjunctival surfaces as a surgical adjunct, MMC has been shown to inhibit fibroblast cell migration, decrease extracellular matrix production, and to induce apoptosis in Tenon's capsule fibroblasts. It is well known that chronic tissue effects from topical MMC administration can persist for many years after the cessation of treatment, similar to the effects of ionizing radiation (Demirci et al 2000; McKelvie and Daniell 2001).

Topical MMC has been reported to cause various histopathologic changes to the conjunctival epithelium. Folberg and associates (1999) reported an analysis of 18 conjunctival biopsy specimens taken from ten patients treated with MMC for PAM with atypia (Salomao et al 1999). The patients in this study were treated with topical $0.02 \%$ MMC for $14-21$ consecutive days, with biopsies being performed 3-6 weeks following the last day of therapy. In these conjunctival specimens, epithelial cells consistently demonstrated dysplastic changes, such as nuclear enlargement and chromatin hyperchromasia, but only in the superficial layers of the conjunctival epithelium. The authors also emphasized that an enlargement of the nucleus and the cell appeared to occur proportionately (ie, cytomegaly), resulting in a preserved nucleus-cytoplasmic ratio. Other histopathologic features included cytoplasmic condensation and eosinophilia, focal epithelial sloughing, and single cell necrosis, all suggestive of apoptosis. The authors commented that the distinction between chemotherapy effect and recurrent neoplasm was less problematic in patients with PAM since the atypical cells following topical MMC were superficially located, while the melanocytes of PAM were in the basal layers of the epithelium. Conversely, degenerative changes in the normal conjunctival epithelium induced by topical MMC may be difficult to distinguish from recurrent or persistent conjunctival squamous neoplasia (Nadjari et al 1999). In a report by Nadjari and colleagues (1999), cytology samples were taken 2 weeks following the completion of topical MMC therapy for conjunctival intraepithelial neoplasia (CIN). Not only were there cellular changes such as chromatin clumping, nuclear pyknosis, and cytoplasmic vacuolization, DNA cytometric analysis revealed aneuploidy in nonneoplastic squamous cells (Nadjari et al 1999). Despite the cellular changes induced by topical MMC treatment, the nucleus to cytoplasmic ratio remained constant in nonneoplastic cells, whereas this ratio increased markedly with recurrent CIN (Nadjari et al 1999; McKelvie and Daniell 2001). McKelvie and Daniell (2001) reported the long-term results of impression cytology following topical MMC therapy for ocular surface squamous neoplasia. Samples from four patients with primary or recurrent ocular surface squamous neoplasia (OSSN) were analyzed between 4 and 17 weeks after topical MMC therapy. The predominant form of cell death in dysplastic cells appeared to be apoptosis, although areas of necrosis were also seen. Apoptotic bodies were identified up to 12 weeks after starting 
topical therapy, although there were fewer numbers of these cells with longer durations of treatment. Dysplastic cellular changes were also seen in the normal epithelial cells, including cytoplasmic vacuolization, cytomegaly, and multiple nuclei or binucleation; these changes persisted up to 8 months after the completion of topical MMC treatment (McKelvie and Daniell 2001). Chronic histopathologic changes to the conjunctival epithelium had also been reported by Demirci and colleagues (2000) in patients treated with topical MMC. In patients who had received between 7-28 days of topical $\mathrm{MMC}$, the authors showed that there was diffuse conjunctival epithelial atrophy and thinning, dyskeratosis, focal keratinization, and stromal fibrosis in areas with atrophic epithelium. The authors noted that these conjunctival changes persisted for an extended period even after the treatment was discontinued, similar to the long-term effects of ionizing radiation (Demirci et al 2000; McKelvie and Daniell 2001).

When prescribing topical MMC for the treatment of conjunctival neoplasms, certain precautions should be taken. Patients and their families are advised to wear latex gloves at all times when handling the medication, and pregnant women should avoid any contact with the drug. MMC is relatively unstable in solution and its action decreases in 8-10 days (Midena et al 2000). Therefore, the formulation for topical MMC needs to be refrigerated and a new bottle dispensed for each week of treatment. Since MMC is a chemotherapeutic agent, all residual bottles should be returned to the pharmacy for proper disposal. Patients should also be instructed to occlude their puncta for at least 5 minutes after the topical instillation of MMC to avoid nasopharyngeal and systemic exposure to the drug.

\section{5-Fluorouracil}

Similar to MMC, topical 5-FU has also been used to inhibit the proliferation of subconjunctival fibroblasts after glaucoma filtration surgery (Katz et al 1995). Several reports have also confirmed the clinical efficacy of topical 5-FU in the treatment of preinvasive ocular surface neoplasia (Yeatts et al 1995, 2000; Midena et al 2000). 5-FU is an antimetabolite used to treat many epithelial cancers because of its actions on rapidly proliferating cells. Within the cell, the drug inhibits thymidylate synthetase during the $\mathrm{S}$ phase of the cell cycle, preventing DNA and RNA synthesis in rapidly dividing cells because of a lack of thymidine. Although the $1 \%$ dose of 5-FU used in clinical studies is somewhat arbitrarily chosen, this dosage appears to be well-tolerated in the vast majority of patients. In addition, the cytologic changes associated with MMC have not been observed with topical
5-FU (de Keizer et al 1986; Yeatts et al 2000). Finally, 5-FU is an inexpensive drug, easily handled by medical personnel and patients, and is stable in aqueous solution for at least 3 weeks. 5-FU also does not require refrigeration, as does MMC (Midena et al 2000).

\section{Alpha-Interferon}

Interferons are a family of glycoprotein molecules that act at cell surface receptors to produce antiviral and antitumor activities through direct and indirect mechanisms (Baron et al 1991; Schechter et al 2002). Systemic interferon-alpha is an established therapeutic agent for hairy cell leukemia, condyloma acuminate, Kaposi's sarcoma in AIDS, and hepatitis (both $\mathrm{B}$ and $\mathrm{C}$ ). Intralesional interferon has also been reported to be effective at treating squamous and basal cell carcinomas of the skin (Fenton et al 2002). Topical IFNapha2b is the recombinant form of interferon-alpha, and this formulation has been shown to be effective for treating conjunctival and corneal intraepithelial neoplasia (CIN) (Vann and Karp 1999). The mechanism of action for treating CIN may be related to an indirect antiproliferative effect of topical interferon on superficial tumor cells, achieved by promoting the immune response through an activation of host cytotoxic effector cells (Baron et al 1991).

\section{Conjunctival neoplasms treated with topical agents Primary acquired melanosis and conjunctival melanoma}

Primary acquired melanosis is a proliferative condition of melanocytes that populate the conjunctival epithelium (Folberg et al 1984, 1985b). Clinically, PAM presents as a superficial flat, brown discoloration of the conjunctiva that most commonly affects middle-aged or older Caucasian patients. The margins of PAM may be indistinct and difficult to identify clinically, particularly if there are variably pigmented or nonpigmented areas. The extent of conjunctival involvement with PAM may also increase and decrease over time. There are two distinct histologic subtypes: PAM without atypia is characterized by benign-appearing melanocytes that populate the basilar epithelium, whereas PAM with atypia is characterized by cytologically abnormal melanocyte subtypes, such as polyhedral cells, spindle cells, large dendritiform cells, or epithelioid cells. The abnormal cells that characterize PAM with atypia may remain restricted to the basilar region or spread to all level of the epithelium. PAM without atypia appears to have minimal malignant potential, whereas 
PAM with atypia may progress to malignant melanoma in up to $46 \%$ of cases (Folberg et al 1984). Cases of PAM with atypia demonstrating certain histopathologic features, such as epithelioid cells or pagetoid extension, carry a 75\%-90\% chance of transforming into invasive melanoma (Jakobiec et al 1989). The median time for PAM with atypia to progress to melanoma is approximately 2.5 years, but it may take as long as 10 years (Folberg et al 1985b). Malignant melanoma of the conjunctiva is commonly associated with PAM with atypia, although it can also arise de novo or from conjunctival nevi (Folberg et al 1985a). The incidence of conjunctival melanoma is only $0.2-0.8$ per million in Caucasian populations (Seregard and Kock 1992; Seregard 1998), but it is a potentially lethal ocular malignancy with an overall 10 year mortality as high as 26\%-30\% (De Potter et al 1993; Seregard 1998).

Currently, surgical resection with cryotherapy applied to the conjunctival margins is the most commonly employed method for treating PAM with atypia. However, using the pigmented margins to define the extent of resection is often problematic due to the diffuse or amelanotic features of these tumors. Local recurrence rates for PAM with atypia after local excision and cryotherapy range between 24\%-79\% with long-term follow-up (Paridaens et al 1994; Shields et al 2001). Recurrences are typically associated with incomplete excision, corneal involvement, and/or multifocal disease. The established management approach for invasive conjunctival melanoma is wide surgical excision, although with long-term follow-up nearly $50 \%-60 \%$ of patients manifest local recurrence (Liesegang and Campbell 1980; Paridaens et al 1994; Demirci et al 2000). Despite orbital exenteration, 33\%-50\% of patients with conjunctival melanomas develop metastatic disease when the thickness of the primary tumor exceeds $1.0 \mathrm{~mm}$ (Paridaens et al 1994).

\section{Melanocytic lesions and MMC}

Over the past decade, there have been numerous papers describing the use of topical MMC for both PAM with atypia and conjunctival melanoma (Finger et al 1993; Frucht-Pery and Pe'er 1996; Demirci et al 2000; Shields et al 2001; Rodriguez-Ares et al 2003; Yuen et al 2003; Kurli and Finger 2005). Finger and colleagues (1993) described the use of topical MMC as a treatment modality for conjunctival melanomas. Since then, his group has published several papers describing their clinical experience with this treatment approach (Demirci et al 2000; Kurli and Finger 2005). In 2005 they published the long-term results of 16 patients with biopsy-proven PAM with atypia (8 patients) or conjunctival
MM (8 patients) treated with topical MMC (Kurli and Finger 2005). When MMC was used as primary therapy, the regimen used in their protocol was one drop of $0.04 \% \mathrm{MMC}$ instilled into the superior conjunctival fornix four times a day for 14 days, discontinuing treatment for 14 days, and then restarting MMC for a second 14 day course of treatment. When given as an adjuvant to surgical excision and cryotherapy, MMC was given for 7 days starting within 2 weeks of surgery. In this study, topical MMC was used as primary therapy in 8 patients with PAM with atypia, in 2 patients with invasive melanoma, and in 6 patients as adjunctive therapy following surgical excision for conjunctival melanoma. Interestingly, all 16 patients in his case series demonstrated a clinical response to topical $\mathrm{MMC}$, defined as regression of the areas of pigmentation. With long-term follow-up, however, five of the ten patients in the primary treatment group developed tumor recurrence: $3 / 8$ patients with PAM with atypia and 2/2 patients with melanoma. For the 6 patients in the adjuvant group, 2 developed tumor recurrences during the follow-up period. Overall, surgical excision was performed in 8 of the 16 cases for tumor recurrence, and 3 cases required orbital exenteration. One of the patients with invasive melanoma treated with MMC as primary therapy had subepithelial nests of melanoma which were resistant to repeated courses of topical treatment. Despite orbital exenteration, this patient ultimately developed metastatic disease 42 months after the initial course of topical MMC. Because of this patient, the authors commented that they were reluctant to use topical MMC as primary treatment for any patient with nodules of invasive melanoma.

Another advocate of topical MMC for conjunctival melanocytic lesions is the group at the Hadassah University Hospital in Jerusalem, Israel (Frucht-Pery and Ilsar 1994; Frucht-Pery and Pe'er 1996; Frucht-Pery et al 2002; Pe'er and Frucht-Pery 2005). Frucht-Pery and Pe'er (1996) successfully treated a 63 year old woman with diffuse PAM with atypia. They treated the patient with two 14 day courses of treatment, the first with $0.2 \mathrm{mg} / \mathrm{ml}$ concentration and the second with $0.4 \mathrm{mg} / \mathrm{ml}$ concentration. The diffuse pigmentation cleared and a repeat biopsy showed no evidence of atypia. A follow-up paper in 2005 described a series of 12 consecutive patients treated by their group with topical MMC for melanocytic lesions of the conjunctiva (Pe'er and Frucht-Pery 2005). Among the 12 patients, 9 had PAM with atypia and 3 had invasive melanoma. The patients in their protocol (except the first patient) used $0.04 \%$ MMC 4 times per day for 2 weeks, with pauses of 2 weeks between courses, until "disappearance or stabilization of the remnants of the pigmentation" 
(Pe'er and Frucht-Pery 2005). MMC was used as primary treatment in all 12 cases and the duration of therapy ranged from $2-5$ cycles. In four patients the pigmentation completely disappeared with MMC treatment. In the other eight patients, some remnants of pigmentation remained, and one of the patients with early invasive melanoma was noted to have a tumor recurrence during the follow-up period and was treated successfully with two more courses of MMC. This series had no patients that required surgical intervention, although the follow-up was shorter than the Kurli series (24 months vs. 81 months) (Kurli and Finger 2005).

Although numerous papers have been published on the use of topical MMC for melanocytic lesions, the total number of patients identified in this review was only 38 (6 studies) (Table 1). As seen in Table 1, MMC $0.04 \%$ was the most commonly used concentration, although $0.02 \%$ was used in one case by Yuen and colleagues (2003), and one case described by Frucht-Pery and Pe'er (1996) was treated with a concentration as high as $0.4 \mathrm{mg} / \mathrm{ml}$. The most commonly used cycle duration was 14 days, with a period of 1-2 weeks between cycles; the total course of treatment was 2-3 cycles in most series. Overall, the clinical response rate appeared to be high, although a successful treatment outcome was defined somewhat differently among clinicians. Some authors defined a successful clinical response as the complete disappearance of pigment and/or negative biopsies after a course of topical treatment. Other authors defined clinical success as either stability of the areas of pigmentation or lack of progression of superficial disease to invasive melanoma. For the purposes of our review, treatment failure was defined as the development of a recurrence, either a new tumor foci or progression of the areas of pigmentation during the period of follow-up. For the group of patients with PAM without atypia treated with topical MMC (as primary treatment), tumor recurrence was noted in 3 out of 22 eyes, for a treatment failure rate of $13.6 \%$. However, note that all 3 cases of PAM recurrence were seen in the study with the longest follow-up (Kurli and Finger 2005), being diagnosed 23, 43, and 54 months after the completion of topical treatment. No patient with PAM with atypia treated with topical MMC was noted to progress to invasive melanoma during the period of follow-up, and none of the patients developed regional or systemic metastasis. When considering conjunctival melanoma, some authors made a distinction between lesions with microscopic invasion of the basement membrane and those with invasive nests of nodular melanoma. For the purposes of our review, we grouped these lesions together since any lesion with an area of invasion through the basement membrane is considered a malignancy. For invasive melanoma, Table 1 shows that 6 out of 16 cases were diagnosed with tumor recurrences, for a failure rate of $37.5 \%$. Although one case was treated with additional topical therapy, the other 5 cases were treated with surgical excision and even exenteration in an attempt to obtain local tumor control. Metastasis was reported in one patient with malignant melanoma who received MMC as primary therapy (Kurli and Finger 2005).

\section{Summary of MMC for melanocytic lesions}

The interpretation of clinical studies on topical MMC is made difficult by the small sample size and variation between the studies in tumor characteristics, previous treatment, length of follow-up, dosing regimen, and whether topical MMC was used as sole therapy, adjuvant, or intraoperative therapy. For PAM with atypia, the success rate of topical MMC compares favorably with the recurrence rate that has been reported following local excision and cryotherapy. Therefore,

Table I Summary of clinical studies of topical MMC used as primary therapy for melanocytic lesions

\begin{tabular}{|c|c|c|c|c|c|c|c|}
\hline Author & DX & No. eyes & Recurred & Surgery & $\begin{array}{l}\text { Follow-up } \\
\text { (months) }\end{array}$ & $\begin{array}{l}\text { MMC dose } \\
(\mathrm{mg} / \mathrm{ml})\end{array}$ & $\begin{array}{l}\text { MMC regimen } \\
\text { (QID/weeks) }\end{array}$ \\
\hline Frucht-Pery and Pe'er 1996 & PWA & 1 & 0 & 0 & 8 & $0.2 \%-0.4 \%$ & 4 \\
\hline Kemp et al 2002 & PWA & 2 & 0 & 0 & $9-16$ & $0.04 \%$ & 4 \\
\hline Yuen et al 2003 & PWA & I & 0 & 0 & 26 & $0.02 \%-0.04 \%$ & 16 \\
\hline Rodriguez et al 2003 & PWA & I & 0 & 0 & 36 & $0.04 \%$ & 4 \\
\hline Kurli and Finger 2004 & PWA & 8 & 3 & 3 & $13-144$ & $0.04 \%$ & $2-4$ \\
\hline Pe'er and Frucht-Pery 2005 & PWA & 9 & 0 & 0 & $6-108$ & $0.04 \%$ & $4-10$ \\
\hline Totals & PWA & 22 & 3 & 3 & & & \\
\hline Rodriguez et al 2003 & MM & I & 0 & 0 & 36 & $0.04 \%$ & 4 \\
\hline Kurli and Finger 2004 & MM & 8 & 5 & 5 & $13-144$ & $0.04 \%$ & $1-4$ \\
\hline Pe'er and Frucht-Pery 2005 & $M M$ & 3 & I & 0 & $6-108$ & $0.04 \%$ & $4-10$ \\
\hline Totals & MM & 12 & 6 & 5 & & & \\
\hline
\end{tabular}

Abbreviations: DX, clinical diagnosis; PWA, primary aquired melanosis with atypia; MM, malignant melanoma; QID, 4x per day. 
topical MMC can be considered a viable therapeutic option for patients with diffuse PAM with atypia diagnosed on incisional biopsy or those patients with positive margins or residual areas of pigment following local excision. In most protocols, MMC 0.04\% has been used 4 times daily for 2 week cycles, with breaks of 1-2 weeks between cycles, and a total of 2-4 cycles to allow for a complete clinical response. It should be emphasized that not all of the areas of pigmentation resolve completely, and a clinical response may continue to be observed for months after the topical treatment has been stopped. Pigment that does not resolve completely after topical MMC therapy should not be considered a treatment failure, since this may represent a grouping of normal melanocytes with increased pigmentation or perhaps extracellular pigment (Pe'er and Frucht-Pery 2005). If a patient has completed an adequate course of therapy but there are residual areas of pigmentation, the lesion should be observed at regular intervals for evidence of progression or re-biopsied. If the area of pigment progresses during the period of followup or the biopsy shows persistent disease, then patients can have a re-treatment with topical MMC if the area is diffuse or local excision if the area is fairly circumscribed.

For invasive conjunctival melanoma, the role of topical MMC in the treatment algorithm is not well-established. Among the 16 patients that have been treated with topical MMC as either primary or adjuvant therapy, 5 cases experienced tumor recurrences $(37.5 \%)$ and 3 patients eventually required orbital exenteration for local tumor control. It was noted by Demirci and colleagues (2000) that nodular nests of subepithelial melanoma appeared resistant to topical MMC. Pe'er and Frucht-Pery (2005) successfully treated 3 patients with conjunctival melanoma using topical MMC as primary therapy, although these three patients had only microscopic invasion on histopathology and one of the patients developed a tumor recurrence. Topical MMC was also used as an adjuvant agent after surgical excision by Finger's group in 6 patients with conjunctival melanomas, and 3 of these patients experienced tumor recurrences. Although the short course of topical therapy in these patients was well-tolerated, the beneficial effect of adjuvant MMC for conjunctival melanomas is questionable (Werschnik and Lommatzsch 2002). Based on the studies that have been published to date, caution is warranted when treating any patient with an invasive conjunctival melanoma with topical MMC as primary treatment. If a conjunctival melanoma is surgically excised and the surgical margins demonstrate an area of PAM with atypia or residual superficial disease of the cornea, topical therapy can be considered a viable alternative to repeat surgical excision.
If the surgical margin is positive for invasive melanoma, surgical excision of the involved area should be strongly considered. This recommendation is particularly relevant for patients with high risk features for metastatic disease, such as location in the caruncle or palpebral conjunctiva, thickness greater than $0.8 \mathrm{~mm}$, previous history of tumor recurrence, or pagetoid growth pattern.

\section{Ocular surface squamous neoplasia}

Lee and Hirst (1995) first proposed the term OSSN to encompass the entire spectrum of dysplastic and carcinomatous lesions of the ocular surface. In its current usage, the term OSSN represents a spectrum of disorders from simple dysplasia to carcinoma in situ to invasive squamous cell carcinoma involving the conjunctiva, limbus, and/or cornea. OSSN lesions involving only the epithelium can also be termed conjunctival or CIN (Pizzarello and Jakobiec 1978). Similar to other mucosal sites, CIN can be classified as mild, moderate, and severe dysplasia based on the extent of involvement; lesions that involve the basal one-third of the conjunctiva are classified as mild, those involving the inner two-thirds are classified as moderate, and lesions that are full thickness are termed severe dysplasia or carcinoma in situ. Invasive disease is most commonly classified as squamous cell carcinoma of the conjunctiva, although more rare variants such as mucoepidermoid and spindle cell carcinoma can be seen (Lee and Hirst 1995). The most common clinical presentation for OSSN is an elevated, vascularized lesion in the limbal region of older male patients who have a history of significant exposure to ultraviolet radiation. Pizzarello and Jokobiec (1978) have described the macroscopic appearance of OSSN as being gelatinous, velvety, papilliform, or leukoplakic. Two other appearances, nodular and diffuse, have also been described (Blodi 1973). Occasionally, OSSN is diagnosed in younger patients with predisposing conditions, such as the human immunodeficiency virus or xeroderma pigmentosum. Human papilloma virus (HPV) is also thought to play a role in the development of OSSN, perhaps in combination with other risk factors such as UV-B exposure. Investigators have detected evidence of active HPV infection by polymerase chain reaction in CIN lesions (Karcioglu and Issa 1997; Scott et al 2002), although it is clear that not all patients infected with HPV develop CIN.

Wide surgical excision has been considered the treatment of choice for OSSN lesions and specialized techniques for decreasing recurrence rates have been described (Shields et al 1997). Despite adjunctive cryotherapy, intraoperative application of alcohol, and other precautions, recurrence rates 
for OSSN following excision range between 15\%-52\% (Lee and Hirst 1995). Incomplete excision with positive surgical margins has been identified as a major risk factor for recurrence. Erie and colleagues (1986) showed that excised lesions with negative surgical margins had a recurrence rate of $5 \%$, whereas the recurrence rate in those lesions that were incompletely excised increased to $53 \%$. Similarly, Pizzarello and Jakobiec (1978) found that if dysplastic tissues were left at the surgical margin, there was a $69 \%$ recurrence rate. The more severe grades of OSSN also appear to recur at higher rates; CIN lesions have a recurrence rate of $17 \%-24 \%$ whereas squamous cell carcinoma recurs in $30 \%-41 \%$ of cases following surgical excision (Erie et al 1986). When adjunctive cryotherapy is used with surgical excision, recurrence rates appear to be reduced, ranging between 7\%-22\% (Divine and Anderson 1983; Dutton et al 1984; Peksayar et al 1989). Cryotherapy is thought to act through its direct destructive effects on cells as well as the obliteration of the microcirculation in the areas treated, resulting in ischemic infarction of abnormal tissues. Other treatment modalities used for OSSN include beta-radiation therapy (Elkon and Constable 1979; Jones et al 1991), gamma radiation (Goldberg et al 1963), immunotherapy with dinitrochlorobenzene (DNCB) (Ferry et al 1976), and the excimer laser for corneal disease (Dausch et al 1994). When considering aggressive treatment options such as enucleation or even exenteration, it should be kept in mind that ocular invasion and systemic metastasis are uncommon occurrences with OSSN (Lee and Hirst 1995).

Over the past ten years, there has been a paradigm shift in the treatment approach of OSSN. Topical agents are now commonly employed for a wide spectrum of OSSN lesions, being used by clinicians as both adjunctive and primary therapy (Frucht-Pery et al 1997; Stone et al 2005). Through the work of various investigators, topical MMC has been established as an effective primary treatment option for OSSN lesions. Rozenman and Frucht-Pery (2000) published a case series of 8 patients who had small, superficial OSSN lesions ( $<8 \mathrm{~mm}$ in diameter) treated with topical MMC as primary therapy. In all eight patients, the size of the CIN started to decrease with one course of therapy with $0.02 \%$ MMC given for 14 days, although in five cases, a second course of $\mathrm{MMC}$ was required to eradicate the lesions. There were also two treatment failures; one case required an excisional biopsy for persistent disease after two courses of $\mathrm{MMC}$, and another patient developed a clinical recurrence 11 months later that was treated successfully with a third course of MMC. This paper was illustrative in that even with small, superficial OSSN lesions, repeated courses of topical $\mathrm{MMC}$ were required to achieve tumor regression. Furthermore, the regrowth of CIN occurred within the first year of treatment with MMC, indicating that some neoplastic cells survived courses of topical therapy despite the observation of "complete" clinical regression. Wilson and colleagues (1997) reported a case series of 7 patients with diffuse CIN who were considered poor surgical candidates or those who had experienced recurrences despite prior surgical treatment. Three patients had moderate to severe dysplasia and four patients had carcinoma in situ. In this protocol, patients used topical MMC $0.04 \%$ for 7 day cycles which were repeated until tumor control was achieved. With this regimen, six of the seven eyes had a complete clinical regression with a maximum of 21 days of therapy, with one patient requiring surgical excision and adjunctive cryotherapy for a partial response. Topical MMC appeared to be effective in this group of patients with more resistant CIN lesions, although a stronger concentration of $0.04 \%$ MMC was employed. Prabhasawat and colleagues (2005) used a lower dose of MMC $(0.02 \%)$, similar to the regimen used by Rozenman, to treat a group of patients with recurrent or extensive OSSN. The patients in this study used $0.02 \%$ MMC continuously 4 times daily until the tumor completely regressed, as observed clinically and proven by impression cytology. This regimen was successful in six of the seven cases, for a success rate of $85.2 \%$. The tumor elimination time ranged between 2-18 weeks and the mean duration of MMC treatment was 5.4 weeks (range, 2-14 weeks). The authors noted that the lower dosage allowed patients to tolerate the therapy for a longer duration than had been reported in the Wilson series, although the total time to tumor elimination was similar to the higher dose interval treatment (Heigle et al 1997; Wilson et al 1997).

Several authors have used topical MMC as a surgical adjunct for OSSN lesions, both preoperatively to decrease the size of diffuse lesions before surgical excision and postoperatively to decrease recurrences (Akpek et al 1999; Frucht-Pery et al 2002; Kemp et al 2002; Chen et al 2004; Shields et al 2005). Shields and colleagues reported their experience using MMC as a neoadjuvant chemotherapy agent for tumor reduction (chemoreduction) prior to the surgical resection of thick, extensive conjunctival SCC (Shields et al 2005). Their protocol involved repeating 7 day treatment cycles until no further regression was evident or the patient became intolerant of the toxic effects of the medication. Surgery was performed at least 2 weeks or more following discontinuation of $\mathrm{MMC}$ to allow for recovery of 
the conjunctiva and adequate wound healing. In this case series, follow-up was 14 months in one case, 22 months in another case, and not mentioned in the 3 rd case. The authors reported that chemoreduction was effective in reducing the tumor base a mean of $57 \%$ using an average of 4 cycles of topical MMC. Postoperative MMC has also been used by several authors as adjunctive therapy to reduce recurrence rates. Chen and colleagues (2004) reported the clinical outcomes of topical MMC used as adjunctive treatment following primary excision of OSSN (Chen et al 2004). In this study, 27 primary OSSN lesions in 26 patients were treated with surgical excision, followed by topical MMC. All cases were treated with one week courses of MMC $0.04 \%$ four times a day, after complete epithelial healing. Nineteen patients received three courses and eight cases received two courses due to ocular toxicity. It was not mentioned whether the surgical margins were positive in these cases. With a mean follow-up of 27 months, there were no tumor recurrences reported in this group, and the authors concluded that MMC treatment following surgical excision was well-tolerated and decreased the recurrence rates of OSSN (Chen et al 2004). Other authors have reported similar outcomes with postoperative MMC following excision of
OSSN lesions, albeit with smaller numbers of cases (Akpek et al 1999; Frucht-Pery et al 2002).

Table 2 summarizes the clinical reports on the use of topical MMC for OSSN. For the purposes of our review, treatment failure was defined as persistent disease despite repeated courses of topical treatment necessitating surgical excision, or clinical evidence of recurrence or regrowth after the last course of topical treatment. When used for superficial disease (ie, dysplasia, carcinoma in situ, recurrent disease), the success rate for topical agents compares favorably to other modalities; overall recurrence rate among 38 patients treated was $24 \%$, with variable lengths of follow-up between the 5 studies with CIN patients. Interestingly, both $0.02 \%$ and $0.04 \%$ concentrations have been used successfully for CIN, although in general, shorter courses were required with the stronger concentration to obtain an adequate clinical response. For invasive SCC, the recurrence rate was only $5 \%$, although there were only 19 patients identified in this review among 5 authors. The case series reported by Frucht-Pery and colleagues (2002) consisted of 5 patients with conjunctival SCC that were referred after incomplete surgical excision. In this series, all 5 patients were shown to have negative conjunctival biopsies after being treated with topical MMC.

Table 2 Summary of clinical studies of topical agents used as primary therapy for OSSN

\begin{tabular}{|c|c|c|c|c|c|c|c|}
\hline Author & Agent-DX & No. cases & $\begin{array}{l}\text { Treatment } \\
\text { failure }\end{array}$ & Surgery & $\begin{array}{l}\text { Follow-up } \\
\text { (months) }\end{array}$ & Dose & $\begin{array}{l}\text { Regimen } \\
\text { (QID/weeks) }\end{array}$ \\
\hline \multirow[t]{2}{*}{ Heigle et al I997 } & $\mathrm{MMC} / \mathrm{SCC}$ & 2 & 0 & 0 & $7-9$ & $0.04 \%$ & 3 \\
\hline & $\mathrm{MMC} / \mathrm{CIN}$ & I & 0 & 0 & 6 & $0.04 \%$ & 3 \\
\hline Wilson et al 1997 & $\mathrm{MMC/CIN}$ & 7 & 1 & I & $2-16$ & $0.04 \%$ & $1-3$ \\
\hline \multirow[t]{2}{*}{ Frucht-Pery et al 1997} & $\mathrm{MMC} / \mathrm{CIN}$ & 16 & 5 & 0 & $3-23$ & $0.02 \%-4 \%$ & $1-4$ \\
\hline & $\mathrm{MMC} / \mathrm{SCC}$ & 1 & 1 & I & 6 & $0.02 \%$ & 4 \\
\hline Rozenman et al 2000 & $\mathrm{MMC/CIN}$ & 8 & 2 & I & $24-44$ & $0.02 \%$ & $2-4$ \\
\hline Shields et al 2002 & $\mathrm{MMC} / \mathrm{SCC}$ & 10 & 0 & 0 & $6-50$ & $0.04 \%$ & $1-4$ \\
\hline Frucht-Pery et al 2002 & $\mathrm{MMC} / \mathrm{SCC}$ & 5 & 0 & 0 & $18-37$ & $0.02 \%-0.04 \%$ & $4-6$ \\
\hline \multirow[t]{2}{*}{ Prabhasawat et al 2005} & $\mathrm{MMC} / \mathrm{CIN}$ & 6 & 1 & 0 & $2-52$ & $0.02 \%$ & $2-14$ \\
\hline & SCC & 1 & 0 & 0 & 33 & $0.02 \%$ & 5 \\
\hline Totals & & 57 & 10 & 3 & & & \\
\hline de Keizer et al 1986 & 5-FU/CIN & 2 & 0 & 0 & & $1 \%$ & \\
\hline \multirow[t]{2}{*}{ Yeatts et al 1995} & 5-FU/CIN & 3 & 0 & 0 & $10-18$ & $1 \%$ & $5-6$ \\
\hline & $5-\mathrm{FU} / \mathrm{SCC}$ & 2 & 2 & 2 & $15-36$ & $1 \%$ & $6-7$ \\
\hline Yeatts et al 2000 & 5-FU/CIN & 7 & 3 & 0 & $7-36$ & $1 \%$ & QID/4-24 d \\
\hline Midena et al 2000 & $5-\mathrm{FU} / \mathrm{SCC}$ & 8 & 1 & 0 & $13-53$ & $1 \%$ & 4 \\
\hline Totals & & 22 & 6 & 2 & & & \\
\hline Maskin 1994 & $\mathrm{IF} / \mathrm{CIN}$ & 1 & 0 & 0 & 9 & $\mathrm{l} \mathrm{m} / \mathrm{ml}$ & 8 \\
\hline Vann and Karp 1999 & $\mathrm{IF} / \mathrm{CIN}$ & 6 & 0 & 0 & 7.2 & $\mathrm{l} \mathrm{m} / \mathrm{ml}$ & $8-20$ \\
\hline Karp et al $200 \mathrm{I}$ & $\mathrm{IF} / \mathrm{CIN}$ & 5 & 1 & 0 & $7-28$ & $\mathrm{l} \mathrm{m} / \mathrm{ml}$ & 16 \\
\hline Schechter et al 2002 & $\mathrm{IF} / \mathrm{CIN}$ & I & 0 & 0 & 19 & $\mathrm{I} \mathrm{m} / \mathrm{ml}$ & 20 \\
\hline Boehm and Huang 2004 & $\mathrm{IF} / \mathrm{CIN}$ & 7 & 2 & 0 & $8-17$ & $\mathrm{l} \mathrm{m} / \mathrm{ml}$ & 12 \\
\hline Totals & & 20 & 3 & 0 & & & \\
\hline
\end{tabular}

Abbreviations: DX, clinical diagnosis; CIN, conjunctival intraepithelial neoplasia; SCC, squamous cell carcinoma; MMC, mitomycin-C; OSSN, ocular surface squamous neoplasia; 5-FU, 5-fluorouracil; IF, interferon; d, days; $\mathrm{m} / \mathrm{ml}$, million units per $\mathrm{ml}$; QID, 4x per day. 
Shields and colleagues (2002) also reported excellent clinical results in ten patients with conjunctival and corneal SCC treated with topical MMC as primary therapy. All of these patients had clinically evident tumors and complete tumor regression was documented in all ten cases; no recurrences have been observed with a mean follow-up of 22 months. In general, the stronger concentration of $0.04 \% \mathrm{MMC}$ was used by the majority of authors for invasive SCC, with a treatment duration ranging between 3-6 weeks.

\section{OSSN and 5-FU}

There are a limited number of studies describing the use of 5-FU for OSSN (de Keizer et al 1986; Yeatts et al 1995; Midena et al 2000). de Keizer and collagues (1986) reported the successful treatment of two patients affected by carcinoma in situ of the conjunctiva and cornea with 1\% topical 5-FU (de Keizer et al 1986). Then Yeatts and colleagues (1995) reported using 5-FU drops to treat six patients with a variety of OSSN lesion: three cases of carcinoma in situ, one case of invasive carcinoma, and two cases of intraepithelial dysplasia. These authors used 5-FU four times daily for 14-21 day cycles with no long-term side effects, although two of the six patients developed tumor recurrences that required surgical excision. Yeatts and colleagues (2000) later published a series of 7 patients with CIN lesions with a minimum of 7 months follow-up. In this study, pulsed dosing of 1\% 5-FU was used, with durations of 2-4 days for each cycle. The number of initial treatment cycles was two to six, with the time between cycles being 30-45 days. Three patients in this series experienced tumor recurrence; two patients were successfully treated with additional cycles of topical 5-FU and one patient required treatment with topical MMC for persistent disease. Finally, Midena and colleagues (2000) reported the results of a prospective, nonrandomized study to evaluate the efficacy of topical 5-fluorouracil (5-FU) in the treatment of recurrent, partially excised, and selected untreated conjunctival SCC. This study had a standardized protocol for following patients and the clinical results were not influenced by concomitant surgery or other treatments. All of the patients in this study had invasive SCC, and they were treated daily for a course of 4 weeks with topical $1 \%$ 5-FU. Cytologic smears were performed at 1, 3, and 6 months. At the last follow-up examination, a conjunctival biopsy was performed at the site of the original lesion, except for two patients who refused any surgery. Patients in the study had a minimum follow up of 13 months, with a range of 13-53 months and a mean of 27 months. Seven patients had complete and stable regression of their conjunctival lesions. One patient had a clinical local recurrence, six months after topical chemotherapy. He was successfully retreated with a second 4 week course of topical 5-FU. Overall, no malignant cells were found after the 4 week course of treatment, except for the recurrent case, on examination of conjunctival cytological smears. In all cases, post-treatment biopsies confirmed the accuracy of these cytological results. In summary, all eight cases with SCC were treated with topical 5-FU alone with remission of the disease after one or more courses of local chemotherapy.

Despite the significant recurrence rate observed in the earlier studies with 5-FU, the Midena paper demonstrated that topical chemotherapy with 5-FU alone was effective in eradicating SCC in these patients without major and/or long term side effects. Although it is difficult to make conclusions based on one report, this pilot study was well-designed and clinicians should be encouraged about the prospect of curing invasive SCC lesions with a 4 week course of topical 5-FU. In addition, 5-FU may have a more favorable side effect profile than topical MMC, although more studies are needed to validate this potential advantage.

\section{OSSN and alpha-interferon}

Between 1993 and 1999, there were encouraging case reports of patients with conjunctival and corneal CIN being treated successfully with interferon (Maskin 1994; Vann and Karp 1999; Schechter et al 2002). Topical interferons for OSSN were first reported to be efficacious in a case reported by Maskin (1994). A subsequent paper by Vann and Karp (1999) described the successful use of a combination of intralesional and topical interferon in six patients with biopsy-proven primary or recurrent CIN. Although the follow-up in these early case reports was only 9-19 months, complete resolution of these lesions was noted, and patients were able to tolerate topical interferon therapy for 2-5 months. Karp and colleagues (2001) have also published a case series of 5 patients treated with topical interferon for corneal and conjunctival intraepithelial neoplasia, 2 of whom were treated for recurrent disease. One patient received almost 4 months of continuous therapy (17 weeks), with clinical resolution noted at 13 weeks. The only patient who experienced a recurrence in this series was treated successfully with a second course of topical interferon which lasted 2 months, and this was followed by another 8 months of maintenance therapy. Boehm and Huang (2004) reported using topical interferon as a single therapeutic agent in the treatment of recurrent corneal and conjunctival intraepithelial neoplasia. All seven patients in this series had suffered recurrences after surgical 
excision, and with topical interferon alone, 5 of 7 patients were successfully treated. Two patients experienced clinical recurrences, noted at 2 months and 1 year of follow-up, and in both cases, topical therapy was re-instituted. The average duration of treatment in this series was 14.1 weeks, and the average period to resolution of disease was 14.5 weeks (range, 5-24 weeks). Ocular side effects were not observed in any of these patients.

\section{Summary of OSSN and topical agents}

For the treatment of both preinvasive and invasive OSSN lesions, topical MMC and 5-FU have been shown to be effective when used as primary therapy. A comparative trial has not been performed between these two agents, and it is therefore difficult to compare clinical results between various series. Topical MMC has a longer track record, and it may prove be more versatile, since it has been used as primary therapy, as a preoperative neoadjuvant agent, and a postoperative therapy to decrease recurrence rates. 5-FU appeared to have slightly higher recurrence rates in earlier studies, but the paper by Midena and colleagues (2000) clearly showed that topical 5-FU was very effective in the primary treatment of invasive SCC. When considering both MMC and 5-FU, recurrences respond quite well to second and third courses of topical treatment, and few patients require surgical intervention. Overall, patients treated with 5-FU may experience fewer side effects than MMC during the treatment course, although permanent ocular toxicity with MMC has not been reported when treating OSSN lesions. Topical interferon may play a role in patients who are intolerant of topical chemotherapeutic agents, although the number of cases treated successfully with this therapy is small and prolonged courses of treatment are required (minimum of 3-4 months).

\section{Pagetoid spread of sebaceous cell carcinoma}

Sebaceous gland carcinoma of the periocular region may present with pagetoid extension of the conjunctiva in $41 \%-80 \%$ of cases (Kass and Hornblass 1989). Despite the common practice of using map biopsies to define the extent of pagetoid extension, surgical eradication of the tumor is complicated by the ill-defined and multifocal nature of the intraepithelial involvement in many patients with sebaceous cell carcinoma (Putterman 1986). Kemp and colleagues (2002) reported treating two patients with pagetoid extension from sebaceous cell carcinoma with topical MMC; in one case MMC $0.04 \%$ was used as a preoperative adjunct before surgical excision, and in the second case, topical
MMC was used both intraoperatively and postoperatively. The second patient developed a clinical recurrence which was treated with another course of topical MMC 0.04\% and a subsequent biopsy 22 months later showed no evidence of disease. Shields and colleagues (2002) presented a series of 3 patients with pagetoid invasion of the conjunctiva treated successfully with topical MMC. After confirmation of disease by map biopsies, patients were started on topical MMC $0.04 \%$ with 7 day cycles; the exact duration of treatment was not reported although the authors commented that treatment courses were repeated until all epithelial disease was judged to be clinically regressed. None of the three patients appeared to suffer from a recurrence, and two of the cases had negative post-treatment biopsies, although the longest follow-up was only 17 months. It should be emphasized that topical MMC should only be used in selected cases of biopsy confirmed sebaceous cell carcinoma as a surgical adjunct for pagetoid extension. In such cases, patients can be treated with topical MMC for residual intraepithelial disease following wide surgical excision of the tumor mass. Caution is also warranted when considering MMC in any patient who has undergone extensive eyelid reconstruction, given that compromised eyelid function is likely to be accompanied by ocular surface abnormalities. It is also important to delay topical treatment until all surgical wounds are healed, to avoid more serious ocular side effects related to wound healing or intraocular penetration of the drug. Tumuluri and colleagues (2007) reported significant corneal complications in a 78 year old man with pagetoid invasion of the conjunctiva treated with topical MMC (Tumuluri et al 2004). Following wide tumor excision and complete upper lid reconstruction, the patient was treated with a total of six weeks of topical MMC $0.02 \%$ four times a day for epithelial disease. Although the patient was recurrence-free at two years of follow-up, his course was complicated by corneal epithelial toxicity and ulceration requiring lateral tarsorrhaphy.

\section{Complications of ocular topical therapy \\ Ocular toxicity of MMC}

Almost all patients treated with topical MMC experience transient side effects, manifesting as conjunctival injection, chemosis, and/or keratitis (Demirci et al 2000; Frucht-Pery et al 2002; Kurli and Finger 2005; Khong and Muecke 2006). In Kurli's series, all patients who were treated for 28 days developed keratoconjunctivitis, which took 4-6 weeks to resolve (Kurli and Finger 2005). It is not uncommon for 
patients to discontinue treatment prematurely due to severe keratoconjunctivitis (Demirci et al 2000). Not surprisingly, patients receiving shorter courses of MMC appear to experience less severe keratoconjunctivitis than those receiving longer courses. Lower concentrations of MMC (0.02\%) also appear to cause fewer side effects (Prabhasawat et al 2005). Therefore, it appears that ocular surface toxicity occurs in a predictable, dosage-dependent pattern. When used as an adjunct to surgical excision, several authors have noted that postoperative $\mathrm{MMC}$ does not prevent wound closure or cause wound dehiscence, if instituted after epithelial healing has occurred (Shields et al 2001). Despite the ocular surface disease, almost all of the patients treated with topical MMC maintain their pretreatment visual acuity (Demirci et al 2000; Pe'er and Frucht-Pery 2005). Permanent ocular side effects with topical MMC therapy are unusual. One of the patients in Kurli's series developed corneal pannus and lost seven lines of vision (Kurli and Finger 2005). Recurrent epithelial defects and/or pannus formation may occur secondary to limbal stem cell deficiency, and caution is warranted in treating patients who have had surgical alteration of this area (Dudney and Malecha 2004). Two patients in Kurli's series also gradually developed cataracts 2-3 years after topical MMC therapy, although direct causation was not proven. Intumescent cataract after topical mitomycin for conjunctival melanomas has been reported, possibly due to intraocular penetration of the drug (Sacu et al 2003). No cases of scleral or corneal melting have been reported, as has occurred following intraoperative application of MMC in glaucoma and pterygium surgery. An intact epithelium probably prevents absorption of these drugs into deeper tissue layers, and therefore topical MMC should not be given to any patient with a corneal or conjunctival epithelial defect. Epiphora secondary to punctal stenosis has been reported (Khong and Muecke 2006), emphasizing the need to place temporary punctal plugs or to have patients to occlude the lower punctum for at least five minutes after instilling the topical mitomycin. Occluding the punctum also minimizes the potential for inducing adverse changes in the nasopharyngeal epithelium with topical MMC therapy.

\section{Ocular toxicity of 5-Fluorouracil}

Although the side effect profile of topical 5-FU has not been extensively studied, clinical studies performed to date have reported no long-term side effects with the ocular administration of this drug. When used continuously for 28 days in the study by Midena and colleagues (2000), transient conjunctivitis and superficial keratitis were observed in all patients during the third or fourth week of treatment. This side effect was easily controlled with topical steroid drops given for 5-6 days. When Yeatts modified the 5-FU protocol into a pulsed dosing regimen, he obtained similar clinical results to the Midena protocol, but no adverse ocular reactions were noted. Scarring of the lacrimal drainage apparatus reported with systemic 5-FU (Shapiro et al 1985), has not been found with topical administration. The cytologic changes in the epithelium associated with MMC have not been observed with topical 5-FU.

\section{Ocular toxicity of interferon}

In general, topical interferon is thought to have fewer side effects compared with other agents. No patient in the study published by Boehm experienced any side effects, despite prolonged use of topical interferon (Boehm and Huang 2004). Schecter and colleagues (2002) reported localized conjunctival injection and folliculosis in 4 of 7 patients treated with topical interferon, which they attributed to the vehicle used in their topical preparation. Kobayashi and colleagues (2002) also reported superficial punctuate keratopathy in one case treated with interferon. Transient fever and muscle pain have been reported as side effects of subconjunctival interferon (Vann and Karp 1999; Kobayashi et al 2002).

\section{References}

Akpek EK, Ertoy D, Kalayci D, et al. 1999. Postoperative topical mitomycin C in conjunctival squamous cell neoplasia. Cornea, 18:59-62.

Baron S, Tyring SK, Fleischmann Jr. WR, et al. 1991. The interferons. Mechanisms of action and clinical applications. JAMA, 266:1375-83.

Blodi FC. 1973. Squamous cell carcinoma of the conjunctiva. Doc Ophthalmol, 34:93-108.

Boehm MD, Huang AJ. 2004. Treatment of recurrent corneal and conjunctival intraepithelial neoplasia with topical interferon alfa $2 \mathrm{~b}$. Ophthalmology, 111:1755-61.

Chen C, Louis D, Dodd T, et al. 2004. Mitomycin C as an adjunct in the treatment of localised ocular surface squamous neoplasia. Br J Ophthalmol, 88:17-18.

Dausch D, Landesz M, Schroder E. 1994. Phototherapeutic keratectomy in recurrent corneal intraepithelial dysplasia. Arch Ophthalmol, 112:22-3.

de Keizer RJ, de Wolff-Rouendaal D, van Delft JL. 1986. Topical application of 5-fluorouracil in premalignant lesions of cornea, conjunctiva and eyelid. Doc Ophthalmol, 64:31-42.

De Potter P, Shields CL, Shields JA. 1993. Malignant melanoma of the conjunctiva. Int Ophthalmol Clin, 33:25-30.

Demirci H, McCormick SA, Finger PT. 2000. Topical mitomycin chemotherapy for conjunctival malignant melanoma and primary acquired melanosis with atypia: clinical experience with histopathologic observations. Arch Ophthalmol, 118:885-91.

Divine RD, Anderson RL. 1983. Nitrous oxide cryotherapy for intraepithelial epithelioma of the conjunctiva. Arch Ophthalmol, 101:782-6.

Donnenfeld ED, Perry HD, Fromer S, et al. 2003. Subconjunctival mitomycin $\mathrm{C}$ as adjunctive therapy before pterygium excision. Ophthalmology, 110:1012-16.

Dudney BW, Malecha MA. 2004. Limbal stem cell deficiency following topical mitomycin $\mathrm{C}$ treatment of conjunctival-corneal intraepithelial neoplasia. Am J Ophthalmol, 137:950-1. 
Dutton JJ, Anderson RL, Tse DT. 1984. Combined surgery and cryotherapy for scleral invasion of epithelial malignancies. Ophthalmic Surg, 15:289-94.

Elkon D, Constable WC. 1979. The use of strontium-90 in the treatment of carcinoma in situ of the conjunctiva. Am J Ophthalmol, 87:84-6.

Erie JC, Campbell RJ, Liesegang TJ. 1986. Conjunctival and corneal intraepithelial and invasive neoplasia. Ophthalmology, 93:176-83.

Fenton S, Kennedy S, Moriarty P. 2002. The role of interferon alpha $2 b$ as an adjunctive treatment in the management of aggressive basal cell carcinoma of the eyelids. Acta Ophthalmol Scand, 80:674-5.

Ferry AP, Meltzer MA, Taub RN. 1976. Immunotherapy with dinitrochlorobenzene (DNCB) for recurrent squamous cell tumor of conjunctiva. Trans Am Ophthalmol Soc, 74:154-71.

Finger PT, Milner MS, McCormick SA. 1993. Topical chemotherapy for conjunctival melanoma. Br J Ophthalmol, 77:751-3.

Folberg R, McLean IW, Zimmerman LE. 1984. Conjunctival melanosis and melanoma. Ophthalmology, 91:673-8.

Folberg R, McLean IW, Zimmerman LE. 1985a. Malignant melanoma of the conjunctiva. Hum Pathol, 16:136-43.

Folberg R, McLean IW, Zimmerman LE. 1985b. Primary acquired melanosis of the conjunctiva. Hum Pathol, 16:129-35.

Frucht-Pery J, Ilsar M. 1994. The use of low-dose mitomycin C for prevention of recurrent pterygium. Ophthalmology, 101:759-62.

Frucht-Pery J, Pe'er J. 1996. Use of mitomycin C in the treatment of conjunctival primary acquired melanosis with atypia. Arch Ophthalmol, 114:1261-4.

Frucht-Pery J, Rozenman Y, Pe'er J. 2002. Topical mitomycin-C for partially excised conjunctival squamous cell carcinoma. Ophthalmology, 109:548-52.

Frucht-Pery J, Sugar J, Baum J, et al. 1997. Mitomycin C treatment for conjunctival-corneal intraepithelial neoplasia: a multicenter experience. Ophthalmology, 104:2085-93.

Goldberg JR, Becker SC, Rosenbaum HD. 1963. Gamma radiation in the treatment of squamous-cell carcinoma of the limbus. Am J Ophthalmol, 55: 811-15.

Heigle TJ, Stulting RD, Palay DA. 1997. Treatment of recurrent conjunctival epithelial neoplasia with topical mitomycin C. Am J Ophthalmol, 124:397-9.

Jakobiec FA, Folberg R, Iwamoto T. 1989. Clinicopathologic characteristics of premalignant and malignant melanocytic lesions of the conjunctiva. Ophthalmology, 96:147-66.

Jones DB, Wilhelmus KR, Font RL. 1991. Beta radiation of recurrent corneal intraepithelial neoplasia. Trans Am Ophthalmol Soc, 89:285-98; discussion 298-301.

Karcioglu ZA, Issa TM. 1997. Human papilloma virus in neoplastic and non-neoplastic conditions of the external eye. Br J Ophthalmol, 81:595-8.

Karp CL, Moore JK, Rosa RH. 2001. Treatment of conjunctival and corneal intraepithelial neoplasia with topical interferon alpha-2b. Ophthalmology, 108:1093-8.

Kass LG, Hornblass A. 1989. Sebaceous carcinoma of the ocular adnexa. Surv Ophthalmol, 33:477-90.

Katz GJ, Higginbotham EJ, Lichter PR, et al. 1995. Mitomycin C versus 5-fluorouracil in high-risk glaucoma filtering surgery. Extended follow-up. Ophthalmology, 102:1263-9.

Kemp EG, Harnett AN, Chatterjee S. 2002. Preoperative topical and intraoperative local mitomycin $\mathrm{C}$ adjuvant therapy in the management of ocular surface neoplasias. Br J Ophthalmol, 86:31-4.

Khong JJ, Muecke J. 2006. Complications of mitomycin C therapy in 100 eyes with ocular surface neoplasia. Br J Ophthalmol, 90:819-22.

Kobayashi A, Yoshita T, Uchiyama K, et al. 2002. Successful management of conjunctival intraepithelial neoplasia by interferon alpha-2b. Jpn J Ophthalmol, 46:215-17.

Kurli M, Finger PT. 2005. Topical mitomycin chemotherapy for conjunctival malignant melanoma and primary acquired melanosis with atypia: 12 years' experience. Graefes Arch Clin Exp Ophthalmol, 243:1108-14
Lee GA, Hirst LW. 1995. Ocular surface squamous neoplasia. Surv Ophthalmol, 39:429-50.

Liesegang TJ, Campbell RJ. 1980. Mayo Clinic experience with conjunctival melanomas. Arch Ophthalmol, 98:1385-9.

Maskin SL. 1994. Regression of limbal epithelial dysplasia with topical interferon. Arch Ophthalmol, 112:1145-6.

McKelvie PA, Daniell M. 2001. Impression cytology following mitomycin $\mathrm{C}$ therapy for ocular surface squamous neoplasia. $\mathrm{Br} J$ Ophthalmol, 85:1115-19.

Midena E, Angeli CD, Valenti M, et al. 2000. Treatment of conjunctival squamous cell carcinoma with topical 5-fluorouracil. Br J Ophthalmol, 84:268-72.

Murphy WM, Soloway MS, Finebaum PJ. 1981. Pathological changes associated with topical chemotherapy for superficial bladder cancer. J Urol, 126:461-4.

Nadjari B, Kersten A, Ross B, et al. 1999. Cytologic and DNA cytometric diagnosis and therapy monitoring of squamous cell carcinoma in situ and malignant melanoma of the cornea and conjunctiva. Anal Quant Cytol Histol, 21:387-96.

Palmer SS. 1991. Mitomycin as adjunct chemotherapy with trabeculectomy. Ophthalmology, 98:317-21.

Paridaens AD, McCartney AC, Minassian DC, et al. 1994. Orbital exenteration in 95 cases of primary conjunctival malignant melanoma. Br J Ophthalmol, 78:520-8.

Paridaens AD, Minassian DC, McCartney AC, et al. 1994. Prognostic factors in primary malignant melanoma of the conjunctiva: a clinicopathological study of 256 cases. Br J Ophthalmol, 78:252-9.

Pe'er J, Frucht-Pery J. 2005. The treatment of primary acquired melanosis (PAM) with atypia by topical Mitomycin C. Am J Ophthalmol, 139:229-34

Peksayar G, Soyturk MK, Demiryont M. 1989. Long-term results of cryotherapy on malignant epithelial tumors of the conjunctiva. $\mathrm{Am} \mathrm{J}$ Ophthalmol, 107:337-40.

Pizzarello LD, Jakobiec FA. 1978. Bowen's disease of the conjunctiva: a misnomer. Birmingham, AL, Aesculapius.

Prabhasawat P, Tarinvorakup P, Tesavibul N, et al. 2005. Topical 0.002\% mitomycin $\mathrm{C}$ for the treatment of conjunctival-corneal intraepithelial neoplasia and squamous cell carcinoma. Cornea, 24:443-8.

Putterman AM. 1986. Conjunctival map biopsy to determine pagetoid spread. Am J Ophthalmol, 102:87-90.

Rodriguez-Ares T, Tourino R, De Rojas V, et al. 2003. Topical mitomycin $\mathrm{C}$ in the treatment of pigmented conjunctival lesions. Cornea, 22:114-17.

Rozenman Y, Frucht-Pery J. 2000. Treatment of conjunctival intraepithelial neoplasia with topical drops of mitomycin C. Cornea, 19:1-6.

Sacu S, Segur-Eltz N, Horvat R, et al. 2003. Intumescent cataract after topical mitomycin-C for conjunctival malignant melanoma. Am $J$ Ophthalmol, 136:375-7.

Salomao DR, Mathers WD, Sutphin JE, et al. 1999. Cytologic changes in the conjunctiva mimicking malignancy after topical mitomycin $\mathrm{C}$ chemotherapy. Ophthalmology, 106:1756-60; discussion, 1761.

Schechter BA, Schrier A, Nagler RS, et al. 2002. Regression of presumed primary conjunctival and corneal intraepithelial neoplasia with topical interferon alpha-2b. Cornea, 21:6-11.

Scott IU, Karp CL, Nuovo GJ. 2002. Human papillomavirus 16 and 18 expression in conjunctival intraepithelial neoplasia. Ophthalmology, 109:542-7.

Seregard S. 1998. Conjunctival melanoma. Surv Ophthalmol, 42:321-50.

Seregard S, Kock E. 1992. Conjunctival malignant melanoma in Sweden 1969-91. Acta Ophthalmol (Copenh), 70:289-96.

Shapiro MS, Thoft RA, Friend J, et al. 1985. 5-Fluorouracil toxicity to the ocular surface epithelium. Invest Ophthalmol Vis Sci, 26:580-3.

Shields CL, Demirci H, Marr BP, et al. 2005. Chemoreduction with topical mitomycin $\mathrm{C}$ prior to resection of extensive squamous cell carcinoma of the conjunctiva. Arch Ophthalmol, 123:109-13.

Shields CL, Naseripour M, Shields JA, et al. 2002. Topical mitomycin C for extensive, recurrent conjunctival-corneal squamous cell carcinoma. Am J Ophthalmol, 133:601-6. 
Shields CL, Naseripour M, Shields JA. 2002. Topical mitomycin-C for pagetoid invasion of the conjunctiva by eyelid sebaceous gland carcinoma. Ophthalmology, 109:2129-33.

Shields CL, Shields JA, Armstrong T. 2001. Management of conjunctival and corneal melanoma with surgical excision, amniotic membrane allograft, and topical chemotherapy. Am J Ophthalmol, 132:576-8.

Shields JA, Shields CL, De Potter P. 1997. Surgical management of conjunctival tumors. The 1994 Lynn B. McMahan Lecture. Arch Ophthalmol, 115:808-15.

Stone DU, Butt AL, Chodosh J. 2005. Ocular surface squamous neoplasia: a standard of care survey. Cornea, 24:297-300.

Tumuluri K, Kourt G, Martin P. 2004. Mitomycin C in sebaceous gland carcinoma with pagetoid spread. Br J Ophthalmol, 88:718-19.

Vann RR, Karp CL. 1999. Perilesional and topical interferon alfa-2b for conjunctival and corneal neoplasia. Ophthalmology, 106:91-7.
Werschnik C, Lommatzsch PK. 2002. Long-term follow-up of patients with conjunctival melanoma. Am J Clin Oncol, 25:248-55.

Wilson MW, Hungerford JL, George SM, et al. 1997. Topical mitomycin $\mathrm{C}$ for the treatment of conjunctival and corneal epithelial dysplasia and neoplasia. Am J Ophthalmol, 124:303-11.

Yeatts RP, Engelbrecht NE, Curry CD, et al. 2000. 5-Fluorouracil for the treatment of intraepithelial neoplasia of the conjunctiva and cornea. Ophthalmology, 107:2190-5.

Yeatts RP, Ford JG, Stanton CA, et al. 1995. Topical 5-fluorouracil in treating epithelial neoplasia of the conjunctiva and cornea. Ophthalmology, 102:1338-44.

Yuen VH, Jordan DR, Brownstein S, et al. 2003. Topical mitomycin treatment for primary acquired melanosis of the conjunctiva. Ophthal Plast Reconstr Surg, 19:149-51. 
